\title{
The Impact of Coronavirus (COVID-19) Related Public-Health Measures on Training Behaviours of Individuals Previously Participating in Resistance Training: A Cross-Sectional Survey Study
}

\author{
James Steele ${ }^{1,2}$ (D) $\cdot$ Patroklos Androulakis-Korakakis ${ }^{1} \cdot$ Luke Carlson $^{3} \cdot$ David Williams $^{4} \cdot$ Stuart Phillips ${ }^{5}$. \\ Dave Smith $^{6}$. Brad J. Schoenfeld ${ }^{7}$. Jeremy P. Loenneke ${ }^{8} \cdot$ Richard Winett $^{9} \cdot$ Takashi Abe $^{8} \cdot$ Stéphane Dufour $^{10}$. \\ Martino V. Franchi ${ }^{11}$. Fabio Sarto ${ }^{11}$. Tommy R. Lundberg ${ }^{12}$. Paulo Gentil ${ }^{13}$. Thue Kvorning ${ }^{14}$. Jürgen Giessing ${ }^{15}$. \\ Milan Sedliak ${ }^{16}$. Antonio Paoli ${ }^{11}$. Fiona Spotswood ${ }^{17}$. Alex Lucas ${ }^{2} \cdot$ James P. Fisher $^{1}$
}

Accepted: 12 February 2021 / Published online: 19 April 2021

(c) The Author(s), under exclusive licence to Springer Nature Switzerland AG part of Springer Nature 2021

\begin{abstract}
Introduction Understanding the impact of lockdown upon resistance training (RT), and how people adapted their RT behaviours, has implications for strategies to maintain engagement in similar positive health behaviours. Further, doing so will provide a baseline for investigation of the long-term effects of these public health measures upon behaviours and perceptions, and facilitate future follow-up study.

Objectives To determine how the onset of coronavirus (COVID-19), and associated 'lockdown', affected RT behaviours, in addition to motivation, perceived effectiveness, enjoyment, and intent to continue, in those who regularly performed RT prior to the pandemic.

Methods We conducted an observational, cross-sectional study using online surveys in multiple languages (English, Danish, French, German, Italian, Portuguese, Slovakian, Swedish, and Japanese) distributed across social media platforms and through authors' professional and personal networks. Adults $(n=5389$; median age $=31$ years [interquartile range $(\mathrm{IQR})=25$, $38]$ ), previously engaged in RT prior to lockdown (median prior RT experience $=7$ years $[\mathrm{IQR}=4,12]$ ) participated. Outcomes were self-reported RT behaviours including: continuation of RT during lockdown, location of RT, purchase of specific equipment for RT, method of training, full-body or split routine, types of training, repetition ranges, exercise number, set volumes (per exercise and muscle group), weekly frequency of training, perception of effort, whether training was planned/ recorded, time of day, and training goals. Secondary outcomes included motivation, perceived effectiveness, enjoyment, and intent to continue RT.

Results A majority of individuals (82.8\%) maintained participation in RT during-lockdown. Marginal probabilities from generalised linear models and generalised estimating equations for RT behaviours were largely similar from pre- to duringlockdown. There was reduced probability of training in privately owned gyms ( 59\% to 7\%) and increased probability of training at home $(\sim 18 \%$ to $\sim 89 \%)$; greater probability of training using a full-body routine $(\sim 38 \%$ to $\sim 51 \%)$; reduced probability of resistance machines ( $66 \%$ to $\sim 13 \%)$ and free weight use ( $96 \%$ to $\sim 81 \%)$, and increased probability of bodyweight training $(\sim 62 \%$ to $\sim 82 \%)$; reduced probability of moderate repetition ranges $(\sim 62-82 \%$ to $\sim 55-66 \%)$ and greater probability of higher repetition ranges ( $\sim 27 \%$ to $\sim 49 \%$ ); and moderate reduction in the perception of effort experienced during-training $(r=0.31)$. Further, individuals were slightly less likely to plan or record training during lockdown and many changed their training goals. Additionally, perceived effectiveness, enjoyment, and likelihood of continuing current training were all lower during-lockdown.

Conclusions Those engaged in RT prior to lockdown these behaviours with only slight adaptations in both location and types of training performed. However, people employed less effort, had lower motivation, and perceived training as less effective and enjoyable, reporting their likelihood of continuing current training was similar or lower than pre-lockdown. These results have implications for strategies to maintain engagement in positive health behaviours such as RT during-restrictive pandemic-related public health measures.
\end{abstract}

Extended author information available on the last page of the article 
Pre-registration https://osf.io/qcmpf.

Preprint The preprint version of this work is available on SportR $\chi \mathrm{iv}$ : https://osf.io/preprints/sportrxiv/b8s7e/.

\section{Key points}

Current physical activity guidelines recommend that adults partake in at least two sessions per week of 'muscle strengthening activity', such as RT, for the major muscle groups of the body.

In early 2020, the onset of the coronavirus (COVID-19) global pandemic and consequent 'social distancing' measures resulted in the temporary, and in some cases extended, global closure of many gyms and leisure facilities; colloquially referred to as 'lockdown'.

Understanding the impact of lockdown upon RT, and how people adapted their RT behaviours, may have implications for strategies to maintain engagement in positive health behaviours such as RT during restrictive pandemic-related public health measures.

Those engaged in RT prior to lockdown appeared mostly able to maintain these behaviours with only slight adaptations in both the location and types of training performed.

However, people trained with lower perceived effort, had lower motivation, perceived training as less effective and enjoyable, and reported they were similarly or less likely to continue with their current training compared with pre-lockdown.

\section{Introduction}

Resistance training (RT) is a well-established method of exercise for promoting health- and fitness-related benefits [1]. Muscle-strengthening through regular practice of RT is universally recognized as an important component of public health guidelines to promote health and some have called for a greater emphasis on RT specifically in their recommendations [2]. Current physical activity guidelines recommend that adults partake in at least two sessions per week of 'muscle strengthening activity', such as RT, for the major muscle groups of the body [3-5]. Recent estimates from self-report data indicate that $\sim 20 \%$ to $30 \%$ meet recommendations for engaging in 'muscle strengthening activities' [6,7]. When RT specifically is considered as the activity, these estimates appear considerably lower [8-12].
Though engagement in efficacious RT is possible in a range of settings and with a variety of modalities [2], commercial gyms, and leisure facilities are a popular option for those seeking to carry out RT programs. These facilities offer a wide range of equipment and amenities not available to most in a home-based setting, thus facilitating engagement. Moreover, the gym environment can foster a sense of camaraderie and motivation, which in turn may positively influence exercise adherence [13]. For example, a study of over 400 adults found that health club members were ten times more likely compared to non-members to meet or exceed muscle-strengthening guidelines [14].

In early 2020, the onset of the coronavirus (COVID-19) global pandemic and consequent 'social distancing' measures resulted in the temporary, and in some cases extended, global closure of many gyms and leisure facilities (amongst other services and amenities as part of wider measures): colloquially referred to as 'lockdown'. As such, millions of gym members were forced to change their exercise habits. Research as to how COVID-19-associated restrictions influenced general physical activity levels is somewhat equivocal, with some studies indicating an increase in overall participation [15-17] and others showing a negative impact [18, 19]. Variances appear to be explained, at least in part, by regionspecific and both demographic and socio-economic factors $[19,20]$. However, while these studies provide insights into general global physical activity patterns, there is little research into how the pandemic influenced RT behaviours which are typically performed in gym settings. While one report suggested that women and younger age groups may have reduced engagement in RT [20], others have reported $\mathrm{RT}$ as being a more common form of exercise during the lockdown [18]. With the closure of the typical locations for engaging in RT, many have offered recommendations to adapt and continue engagement in 'muscle strengthening activity' such as RT at home, and with alternative modalities (such as bodyweight exercise or home equipment), both for the general population [21-25] and for sports participants [26-28].

Understanding the impact of lockdown upon RT, and how people adapted their RT behaviours, is expected to have implications for strategies to maintain engagement in positive health behaviours such as this during-restrictive pandemic-related public health measures. Further, doing so will provide a baseline for investigation of the long-term effects of these measures upon behaviours and perceptions and facilitate future follow-up study. Therefore, the purpose 
of this study was to determine how the onset of COVID19 , and the associated lockdown, affected RT behaviours, in addition to motivation, perceived effectiveness, enjoyment, and intent to continue, in those who regularly performed RT prior to the pandemic.

\section{Methods}

\subsection{Study Design}

An online cross-sectional survey study was conducted during April and May 2020 during which time a number of governments worldwide had implemented lockdown strategies in an effort to contain the spread of COVID-19. The initial phase of this overall project, which also includes qualitative data capture (currently ongoing) and longitudinal follow-up scheduled to be conducted in April and May 2021, sought to examine the immediate impact of lockdown upon the RT behaviours of those who were regularly engaged in such behaviours prior to lockdown. Intention to conduct this study was pre-registered on the Open Science Framework (https://osf.io/qcmpf). Where deviations from the originally pre-registered protocol occurred, these are detailed in this manuscript. This study received ethical approval from the Health, Exercise, and Sport Science Ethics Committee at Solent University (fishj2020).

\subsection{Sampling and Population}

Surveys were conducted in two stages: first, an English language survey was launched on April $23^{\text {rd }} 2020$; this was then followed up by the launch of translated versions of the survey (Danish, French, German, Italian, Portuguese, Slovakian, Swedish, and Japanese) on April 29th and 30th 2020. All surveys remained open until May 15th 2020. The survey was primarily distributed via hyperlink across social media platforms and through a number of the authors' professional and personal networks.

Our original target sample size was $n=2606$ based upon two considerations that are detailed in the pre-registration: (1) that required for detection of a 'small' effect size at a power of $\beta=0.80$, and an adjusted $\alpha=0.004$ for the number of dependent variables originally pre-registered ${ }^{1}(14$ questions relating to RT behaviours); and (2) based on this

\footnotetext{
${ }^{1}$ Notably, our original analysis approach was incorrect as some aspects of the measures used were overlooked in the haste with which the project was set-up and launched in response to the implementation of lockdown measures. We detail these changes below, though due to the final sample size yielded we are confident that, under the assumptions of the data generating process and model used, our results offer sufficient power for any tests, and a high degree of precision for interval estimates.
}

yielding an acceptable precision of estimate for survey responses of $2 \%$ margin of error at the $95 \%$ confidence level, based upon estimated worldwide participation rates for RT.

Across the nine surveys a total of 18,862 respondents accessed the open links to the survey, with 7947 completing all required questions. Participants were provided with an information sheet containing all details of the study and then were required to confirm they had understood this and provide informed consent to participate. We implemented a number of screening criteria to yield a final data set to be included in analysis. Those who did not meet the inclusion criteria (self-reported as previously engaged in $\mathrm{RT} \sim 2 \times /$ week in line with current physical activity guidelines [3-5] for at least 6 months prior to lockdown, and if their usual training facility was currently inaccessible AND/OR they were engaging in social distancing due to government recommended/implemented measures ${ }^{2}$ ) were excluded, as were those failing the attention check included in the survey or any responses to the question designed to screen for bots. We also removed participants with out of range responses ${ }^{3}$ for any continuous open field variables in the survey. ${ }^{4}$ The final sample size included in the analysis was $n=5389$.

\subsection{Survey Implementation and Measures}

The surveys were administered through JISC Online Surveys (Bristol, UK). All surveys are available in the supplementary files (S2). Further, we have setup a dummy version of the online survey so that readers can access it to see exactly how questions were presented to respondents (https://solent.onlin esurveys.ac.uk/covid-19_rt_survey_copy). In brief, the survey was designed to take no longer than $\sim 10 \mathrm{~min}$ to complete and asked participants to provide general demographic and

\footnotetext{
${ }^{2}$ Due to the known variation in both timing of the pandemic's effects, and the implementation of containment measures, across different countries we screened prior to inclusion such that only individuals who were currently impacted by these measures were included. Based upon survey timings respondents had therefore been under lockdown conditions including closures of their usual training facility AND/OR social distancing for $~ 3-5$ weeks.

3 The limits set can be seen in the analysis code available in supplementary file (S1).

${ }^{4}$ An exception to this was for income. We asked respondents to provide their annual household incomes in the currency appropriate based on their country of residence, followed by selecting from a list of currency codes the one used. We then used currency exchange rates (as of 31st July 2020) to convert all values to EUR. However, this yielded a high proportion of very high values (860 respondents with annual household incomes above 1 million EUR) leading us to question whether these questions had been understood fully. Thus, we chose not to filter by this variable due to concerns regarding the quality of this data. We plan to attempt to address this during-follow up and obtain data that might be used for retrospective examination of income effects.
} 
personal details, followed by $15^{5}$ questions relating to different aspects of their RT behaviours prior to implementation of lockdown measures. These included questions regarding the following:

1. Where participants undertook resistance training.

2. Whether they purchased specific equipment for resistance training.

3. How they trained i.e., alone, with a partner, supervised, etc.

4. Whether they performed full body or split routines.

5. The types of training they performed.

6. The repetition ranges used.

7. Number of exercises per workout.

8. The set volumes per exercise.

9. The set volume per muscle group.

10. The frequency per week they trained.

11. The rating of perception of effort.

12. The current goals for training.

13. Whether they planned their workouts.

14. Whether they recorded their workouts.

15. The time of day they trained.

Participants were then asked if they were currently engaging in RT in addition to their current motivation to train. Those who were still engaged in RT were then prompted to complete the same 15 questions relating to RT behaviours, in addition to questions comparing their current training to their training prior to lockdown regarding its perceived effectiveness, enjoyment, and the likelihood they would continue with their current training program. Participants were also offered the opportunity to complete an open field to provide additional information regarding their answers, and were invited to be contacted to participate in both follow-up surveys as well as semi-structured interviews. The questions relating to RT behaviours were a combination of single-item response category variables (SRCVs, e.g. yes/ no questions), multiple response category response variables (MRCVs, i.e. please select all that apply ${ }^{6}$ ), and one ordinal variable provided on an 11-point (0-10 point) scale (this was for perception of effort during-RT and the scale used was adapted from one previously developed by Steele et al. [29] available at: https://osf.io/ufvy8/). All survey questions were compulsory and so complete data were available for all those who finished the survey.

\footnotetext{
5 Note, the pre-registration reported that 14 questions were to be included. However, prior to launch yet after pre-registration, we added an additional question.

${ }^{6}$ We opted to use MRCVs for RT variables to allow for individuals who might vary their routines from week to week (i.e. they may engage in what is referred to as 'periodization') to respond without feeling the need to arbitrarily select a particular category.
}

\subsection{Statistical Analysis}

\subsubsection{Main Analysis}

The main analysis examined the effects of lockdown upon respondents' self-reported RT behaviours. ${ }^{7}$ For all outcomes, those who reported having stopped participating in RT had their responses coded as NAs (in essence dropped, i.e., not applicable) for the during-lockdown answers. Thus, we report the proportion of those who reported having stopped participation, and then the main analysis is constrained to examining the change in specific RT behaviours from pre- to during-lockdown.

SRCVs were examined using binomial logistic regression upon response $(0=$ no, $1=$ yes $)$ examining the withinrespondent fixed effect of 'time' (i.e., pre- and duringlockdown; time $=1$, and time $=2$, respectively). MRCVs' responses $(0=$ no, $1=$ yes $)$ were similarly examined with the within-respondent fixed effect of 'time' (i.e. pre- and during-lockdown; time $=1$, and time $=2$, respectively), yet with the addition of 'item' as a fixed factor, and the interaction of 'item $x$ time' allowing us to examine how responses to items changed over time. For MRCVs a generalised estimating equations (GEE) approach was used as suggested by Suesse and Liu [32] for analysis of repeated MRCVs; however, we were able to apply unstructured covariance specification to the model, due to the size of our sample, thus yielding the best model fit. In essence, this GEE approach meant we did not make any assumptions about subject-specific joint distributions. Instead, it permitted identification of correlation structures over both time and between items within each MRCV. Unique Respondent Number (URN; i.e. 'case') was specified as the cluster. From these models we extracted and present the predicted marginal probabilities of a positive response (i.e. $1=$ yes) for a given SRCV or item within a MRCV along with their model specific 95\% compatibility (confidence) intervals. This was chosen over the

\footnotetext{
7 As noted, the analysis presented here differs from that which was pre-registered. Due to the haste of project set-up in light of the suddenness of implementation of lockdown measures we overlooked some aspects of the data that would be generated. This included the paired nature of comparisons for SRCVs, and assumptions of dependency between items underlying the MRCVs. Thus, we have ignored our simple original pre-registered analysis plan aimed at testing the hypothesis of marginal independence. Instead, in part due to the sample size achieved which we anticipated would have yielded low $p$ values with relative lack of information regarding the meaningfulness of the findings, we have analysed and presented our data using an alternative modelling strategy. We have also opted to avoid dichotomizing the existence of an effect for the main results and therefore did not employ traditional null hypothesis significance testing, which has been extensively critiqued $[30,31]$. Instead, we consider the implications of all results compatible with these data, from the lower limit to the upper limit of the interval estimates, with the greatest interpretive emphasis placed on the point estimate.
} 
presentation of model coefficients as log-odds or odds ratios given that most people find these unintuitive to interpret with many mistaking the latter for probability or risk anyway. These probabilities were then qualitatively interpreted and described. Supplementary model summary tables are available in the supplementary files (S3) including all predictors' log-odds estimates, 95\% compatibility (confidence) intervals, and $p$ values (note, for MRCVs the reference category for 'item' is always the first item on the question based on how it was presented to respondents).

The lone ordinal variable (rating of perceived effort) was analysed comparing pre- and during-lockdown using an asymptotic Wilcoxon test from which a standardised effect estimate was produced. Cohen's $r$ was calculated as $\sqrt{\frac{Z}{n}}$ to examine the magnitude of the standardised effect; however, data are also visually presented with a scatterplot in the raw scale units presenting points with a small jitter thus showing the density of pairings of pre-during scores.

All analyses were conducted using $\mathrm{R}$ (version 3.6.1; R Core Team, 2019) and RStudio (version 1.2.1335; RStudio Team, 2018). GEE models were produced using the package 'geepack' [33], interaction plots for MRCVs were produced using the package 'sjplot', and all other data visualisation was produced using 'ggplot'.

\subsubsection{Exploratory Analyses}

Analyses performed in an exploratory manner were conducted through visualisation of the dataset. We highlight only those exploratory findings that appear noteworthy in this manuscript.

First, we explored the relationships between demographic characteristics with RT behaviours over time, in addition to whether respondents continued training (for continuation of training we also examined current motivation as a predictor), by examining the naïve probabilities of engaging in RT behaviours over these predictors. For continuous demographic predictors we plotted smoothed conditional means using loess in 'ggplot' and for categorical demographic predictors we plot means with compatibility (confidence) intervals produced using basic nonparametric bootstrap sampling from the 'Hmisc' package.

Second, we explored the impact of RT behaviours engaged in during-lockdown upon perceived effectiveness of training, enjoyment from training, and likelihood of continuing training. For SRCVs and MRCVs we present ridgeplots using 'ggridges' showing the distributions of perceived effectiveness of training, enjoyment from training, and likelihood of continuing training across each of the question items as predictors. For the lone ordinal variable (rating of perceived effort) we plotted smoothed conditional means using loess.

\section{Results}

The final number of respondents after filtering for each survey is provided in Table 1 in addition to the percentage of the total respondents. Full details of this process, numbers at each stage of the survey, and a separate table of respondents countries are available in the supplementary materials (S4) (Table 2).

\subsection{Main Results: Effects of Lockdown on RT Behaviours}

As noted, model-specific summary tables are available in the supplementary files. Here we focus on reporting the key findings. As intended, and due to the high precision of estimates for predicted marginal probabilities ${ }^{8}$ from the models (see figures below ${ }^{9}$ ) we emphasise the point estimates and their magnitude in our reporting.

\subsubsection{Are You Currently Engaged in RT?}

Of the complete sample of 5389 respondents, 4466 (82.8\%) reported to still be engaging in some form of RT during-lockdown.

\subsubsection{Where Did You Usually Undertake RT?}

The GEE results of this MRCV question are presented in Fig. 1. As can be seen clearly, prior to lockdown the greatest probability by a large margin was for individuals to be training in a 'privately owned gym' ( $59 \%$ probability $)$ with roughly similar probabilities given to all other options comparatively speaking (all $20 \%$ or lower). However, duringlockdown there was a considerable increase in the probability of training at 'home' ( $\sim 89 \%$ probability) and also a slight increase in the probability of training in a 'public park' (from 8\% to 15\%). With the exception of 'other' all remaining options dropped to $<5 \%$ probability, though there was a slightly elevated probability of training in a 'privately owned gym' still at during-lockdown ( $\sim \%$ probability), perhaps influenced by those who were gym owners themselves?

\footnotetext{
8 The predicted probabilities can be easily interpreted by considering an example population of $n=100$. If the probability of some behavior or event is 0.75 (or $75 \%$ ) for example then this would mean that 75 out of 100 individuals would be engaging in the behavior or experience the event. This may aid readers in interpreting the probabilities presented.

9 All figures include corresponding 95\% compatibility (confidence) intervals for estimates from the models yet in some figures they are so precise that they appear subsumed in the point estimate on the display.
} 
Table 1 Responses to surveys

\begin{tabular}{lll}
\hline Survey language & Total responses & $\begin{array}{l}\text { Percentage of } \\
\text { total responses } \\
(\%)\end{array}$ \\
\hline Danish & 97 & 2 \\
English & 3589 & 67 \\
French & 37 & 1 \\
German & 196 & 4 \\
Italian & 639 & 12 \\
Japanese & 133 & 2 \\
Portuguese & 421 & 8 \\
Slovakian & 64 & 1 \\
Swedish & 213 & 4 \\
Total & 5389 & 100 \\
\hline
\end{tabular}

\subsubsection{Have You Previously Purchased Specific Equipment (Including Weights, Machines, Bands, or Other Devices) to Support Your RT?}

The binomial logistic regression results of this SRCV question are presented in Fig. 2. Individuals were more likely to have purchased specific equipment prior to lockdown $(\sim 70 \%$ probability) as compared to during-lockdown ( $48 \%$ probability).

\subsubsection{How Did You Usually Train?}

The GEE results of this MRCV question are presented in Fig. 3. The pattern of probabilities across items was largely similar both pre- and during-lockdown with a considerably higher probability that individuals would be training 'alone' compared with all other options. Notable changes, however, include slight reductions in both training 'supervised in person' (from 15\% to 2\%) and 'with a partner' (from 32\% to $21 \%$ ). There were slight increases in both training 'supervised virtually/online' and 'video training' but the probabilities of these responses remained low ( 5-6\% probability).

\subsubsection{Did You Usually Perform a Full Body or Split Routine?}

The binomial logistic regression results of this SRCV question are presented in Fig. 4. Compared with prior to lockdown ( $38 \%$ probability), individuals were more likely to be using full-body routines during-lockdown $(\sim 51 \%$ probability).

\subsubsection{What Types of Training Did You Usually Perform?}

The GEE results of this MRCV question are presented in Fig. 5. Prior to lockdown,individuals had the greatest probability of utilising 'free weights' ( 95\% probability) with relatively similar probabilities of using 'resistance machines' ( $\sim 66 \%$ probability), 'bodyweight exercise' ( $\sim 61 \%$ probability), and performing 'cardio/aerobic exercise' ( 55\% probability). During lockdown, there was both a slight drop in the probability of using 'free weights' ( $\sim 80 \%$ probability) and a slight increase in the probability of using 'bodyweight exercise' ( $\sim 83 \%$ probability). Finally, there was a considerable decrease in the probability of training with 'resistance machines' ( 13\% probability).

\subsubsection{What Repetition Range/Exercises/Set Volumes Did You Usually Use?}

The GEE results of these four MRCV questions are presented in Fig. 6. Overall, the patterns for these are comparable from pre- to during-lockdown. An exception to this appears to be with respect to the repetition ranges used with a reduction in the probability of using lower repetition ranges (' $1-5$ reps' and ' $6-10$ reps') and an increase in the probability of using very high repetitions ('> 15 reps').

\subsubsection{How Often Per Week Did You Usually Train?}

The GEE results of this MRCV question are presented in Fig. 7. Overall, the patterns for frequency of training are comparable from pre- to during-lockdown with only a slight yet notable reduction in the probability of training ' $4-5 \times$ / week' (from $\sim 50 \%$ to $40 \%$ probability).

\subsubsection{What Was Your Usual Intensity of Perceived Effort in Your Training?}

The Wilcoxon signed-rank test yielded a $Z$ statistic of 32.313. Rating of perceived effort was reduced from pre$($ median $=8)$ to during-lockdown (median $=7)$. The effect size for this difference was 'moderate' $(r=0.31)$. Figure 8 shows paired responses for pre- and during-lockdown rating of perceived effort scores. The vertical and horizontal lines for median scores and identity reference line show that scores were lower during-lockdown with the majority of data points falling in the bottom right portion of the plot.

\subsubsection{Did You Usually Plan/Record Each Workout?}

The GEE results of these two MRCV questions are presented in Fig. 9. Overall, the patterns for these are comparable from pre- to during-lockdown with individuals more likely to plan, but not record, their workouts and only a slight reduction in these probabilities during-lockdown. 
Table 2 Descriptive demographics of final sample

\begin{tabular}{|c|c|}
\hline Characteristic & $n=5389^{\mathrm{a}}$ \\
\hline Age (years) & $31(25,38)$ \\
\hline \multicolumn{2}{|l|}{ Sex } \\
\hline Female & $2023(38 \%)$ \\
\hline Male & $3366(62 \%)$ \\
\hline $\mathrm{BMI}\left(\mathrm{kg} \mathrm{m}^{2}\right)$ & $24.9(22.8,27.4)$ \\
\hline \multicolumn{2}{|l|}{ Race } \\
\hline Asian & $316(5.9 \%)$ \\
\hline Black & $78(1.4 \%)$ \\
\hline Mixed & $277(5.1 \%)$ \\
\hline Other & $135(2.5 \%)$ \\
\hline Prefer not to disclose & $36(0.7 \%)$ \\
\hline White & $4547(84 \%)$ \\
\hline \multicolumn{2}{|l|}{ Development } \\
\hline Other & $14(0.3 \%)$ \\
\hline Rural & $574(11 \%)$ \\
\hline Suburban & $2076(39 \%)$ \\
\hline Urban & $2725(51 \%)$ \\
\hline \multicolumn{2}{|l|}{ Employment } \\
\hline Employed full time & $2529(47 \%)$ \\
\hline Employed part time & $530(9.8 \%)$ \\
\hline Furloughed & $358(6.6 \%)$ \\
\hline Other & $600(11 \%)$ \\
\hline Self-employed & $891(17 \%)$ \\
\hline Unemployed & $481(8.9 \%)$ \\
\hline \multicolumn{2}{|l|}{ Working-Environment } \\
\hline No & $792(15 \%)$ \\
\hline Yes, but I am still able to work from my usual location & $1021(19 \%)$ \\
\hline $\begin{array}{l}\text { Yes, I am currently not working due to being furloughed by my employer, or due to a reduction in freelance work if self } \\
\text { employed }\end{array}$ & $1245(23 \%)$ \\
\hline Yes, I am now working remotely (I was previously based at a non-home location all or most of the time) & $2331(43 \%)$ \\
\hline Self-isolation & $1133(21 \%)$ \\
\hline Children & $1181(22 \%)$ \\
\hline \multicolumn{2}{|l|}{ Number of children } \\
\hline 0 & $2(0.2 \%)$ \\
\hline 1 & $527(45 \%)$ \\
\hline 2 & $487(41 \%)$ \\
\hline 3 & $129(11 \%)$ \\
\hline 4 & $30(2.5 \%)$ \\
\hline 5 & $4(0.3 \%)$ \\
\hline 6 & $2(0.2 \%)$ \\
\hline Unknown & 4208 \\
\hline Care-giver & $228(4.2 \%)$ \\
\hline Education & $1938(36 \%)$ \\
\hline RT-experience (years) & $7(4,12)$ \\
\hline Strength-sports & $1010(19 \%)$ \\
\hline Bodybuilding & $449(8.3 \%)$ \\
\hline Endurance-sports & $1666(31 \%)$ \\
\hline Other-sports & $2631(49 \%)$ \\
\hline
\end{tabular}

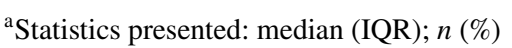




\subsubsection{Did You Usually Train in the Morning, Afternoon, Evening or Night-Time?}

The GEE results of this MRCV question are presented in Fig. 10. Overall, the patterns for what time of day individuals usually trained are comparable from pre- to duringlockdown with only a slight, yet notable, increase in the probability of training in the 'afternoon' (from 43 to 52\% probability).

\subsubsection{What Were Your Goals from Participation in Training?}

The GEE results of this MRCV question are presented in Fig. 11. Prior to lockdown the most prominent goal was 'strength' $(\sim 83 \%)$, whereas during-lockdown this was 'general health' ( 77\%). Further, a small majority (54\%) reported to have maintained the same goals from pre- to during-lockdown.
Fig. 1 Predicted marginal probabilities for "Where did you usually undertake RT?" over 'time' (i.e. pre- and duringlockdown) and 'items'. Note: error bars are $95 \%$ compatibility (confidence) intervals; $Y$ axis is ordered by the magnitude of probabilities at 'pre-lockdown'

Fig. 2 Predicted marginal probabilities for "Have you previously purchased specific equipment (including weights, machines, bands, or other devices) to support your RT?" over 'time' (i.e. pre- and duringlockdown). Note: error bars are 95\% compatibility (confidence) intervals
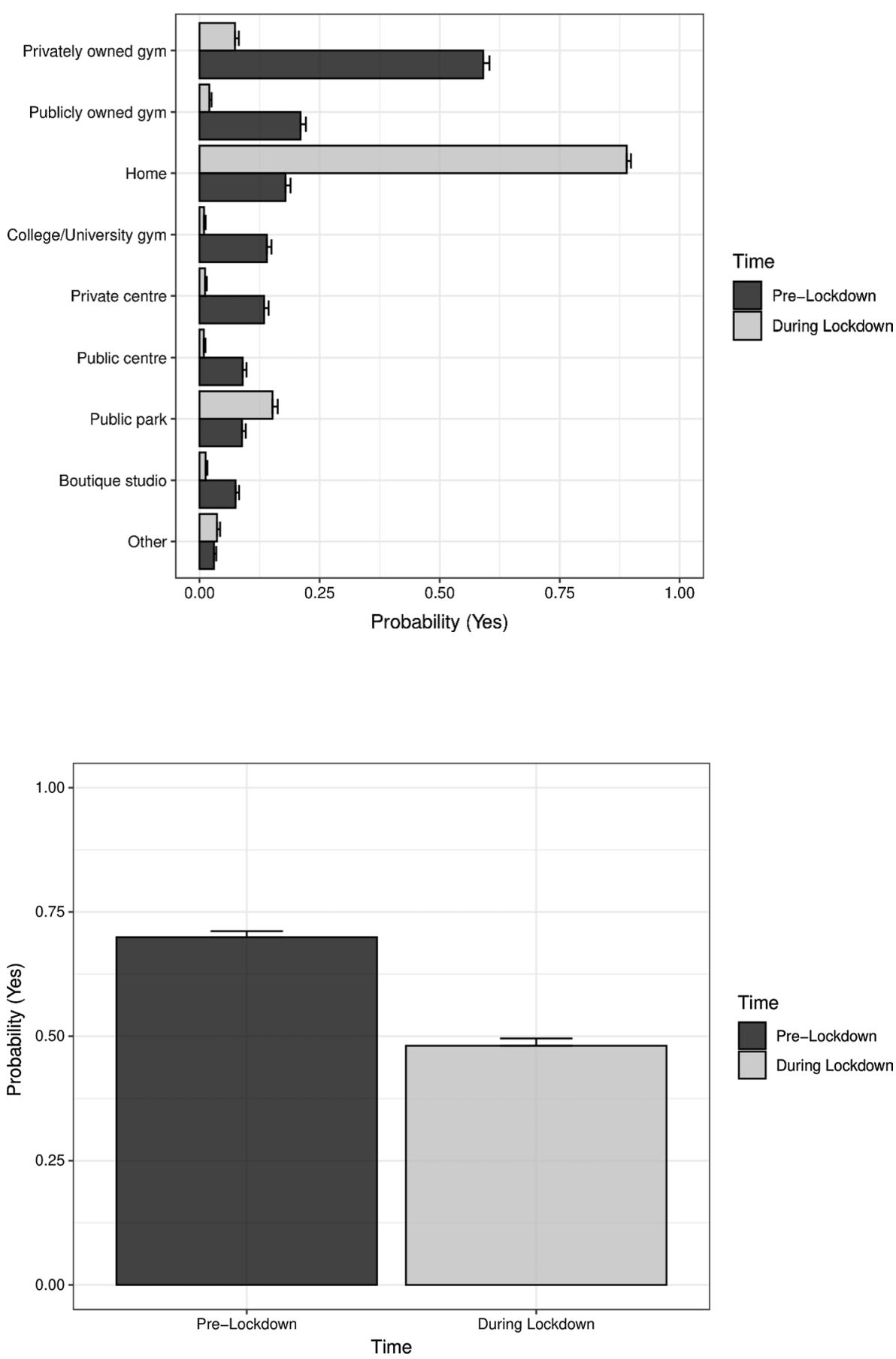
Fig. 3 Predicted marginal probabilities for "How did you usually train?" over 'time' (i.e. pre- and during-lockdown) and 'items'. Note: error bars are 95\% compatibility (confidence) intervals; $\mathrm{Y}$ axis is ordered by the magnitude of probabilities at 'pre-lockdown'
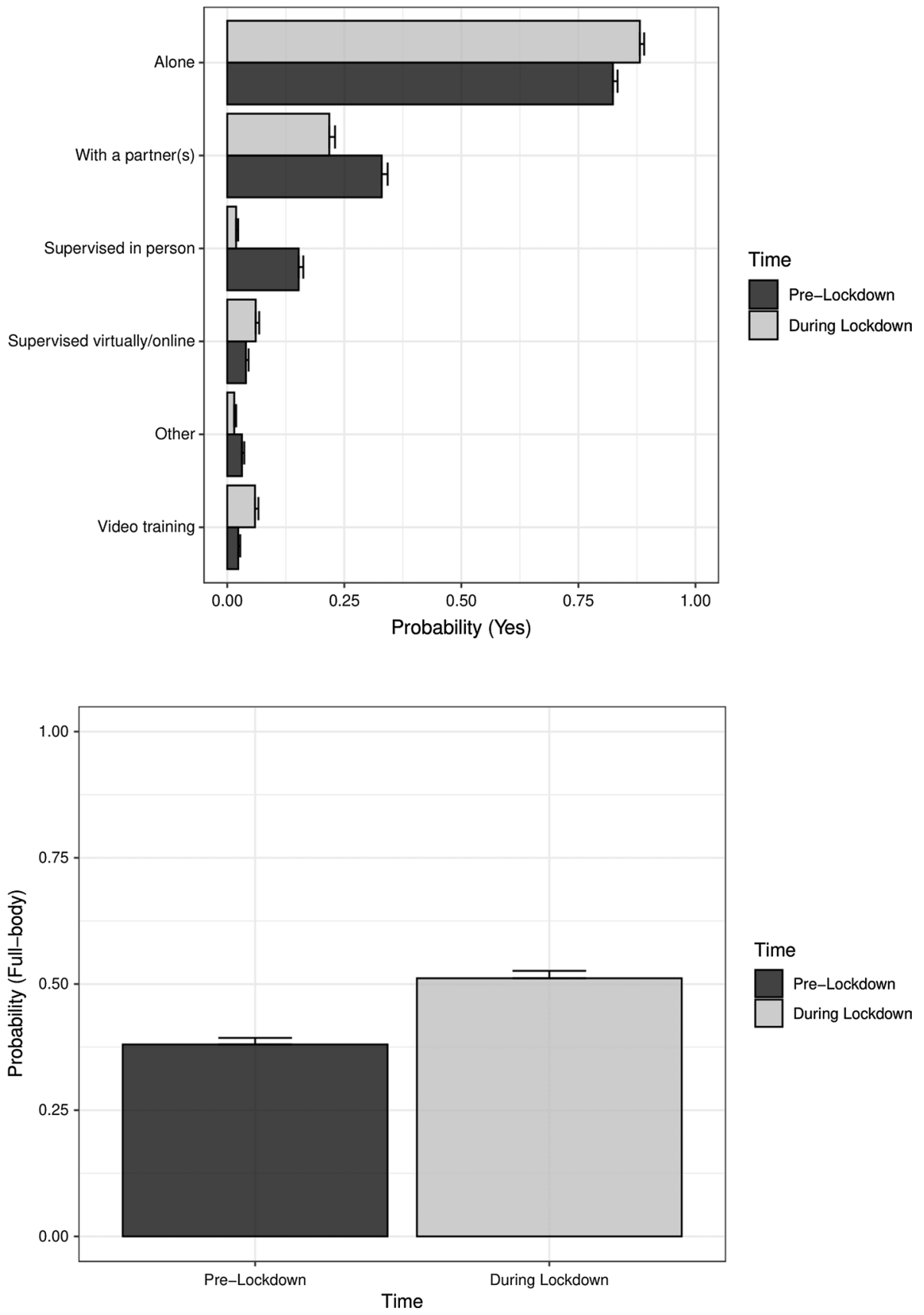

Fig. 4 Predicted marginal probabilities for "Did you usually perform a full body or split routine?" over 'time' (i.e. preand during-lockdown). Note: error bars are $95 \%$ compatibility (confidence) intervals

\subsection{Secondary Outcomes and Exploratory Analyses}

\subsubsection{Motivation, Enjoyment, and Likelihood of Continuing Current Training}

Frequencies and percentages for questions relating to respondents' current motivations, enjoyment, and likelihood of continuing with current training are shown in Fig. 12. For the whole sample, individuals had mostly 'similar' or 'lower' (both 33\%) current motivation to train, and for those who had continued training there was notably 'lower' enjoyment (40\%). However, most people who continued training said they were 'similarly likely' to continue with their current training $(41 \%)$.

\subsubsection{Perceived Effectiveness}

Frequencies and percentages for questions relating to respondents' perceived effectiveness of their current training, with a breakdown based upon whether goals had changed or not, are shown in Fig. 13. For those whose goals remained the same a majority indicated they felt that their current training was 'similarly effective' (44\%), or 
Fig. 5 Predicted marginal probabilities for "What types of training did you usually perform?" over 'time' (i.e. pre- and during-lockdown) and 'items'. Note: error bars are 95\% compatibility (confidence) intervals; $y$ axis is ordered by the magnitude of probabilities at 'pre-lockdown'


Fig. 6 Predicted marginal probabilities for "What repetition range/ exercises/set volumes did you usually use?" over 'time' (i.e. pre- and during-lockdown) and 'items'. a shows the repetition range used, b shows the number of exercises used, $\mathbf{c}$ shows the number of sets per exercise used, and $\mathbf{d}$ shows the number of sets per muscle group used. Note: error bars are 95\% compatibility (confidence) intervals; $y$ axis is ordered by the magnitude of probabilities at 'pre-lockdown' 
Fig. 7 Predicted marginal probabilities for "How often per week did you usually train?" over 'time' (i.e. pre- and duringlockdown) and 'items'. Note: error bars are $95 \%$ compatibility (confidence) intervals; $Y$ axis is ordered by the magnitude of probabilities at 'pre-lockdown'
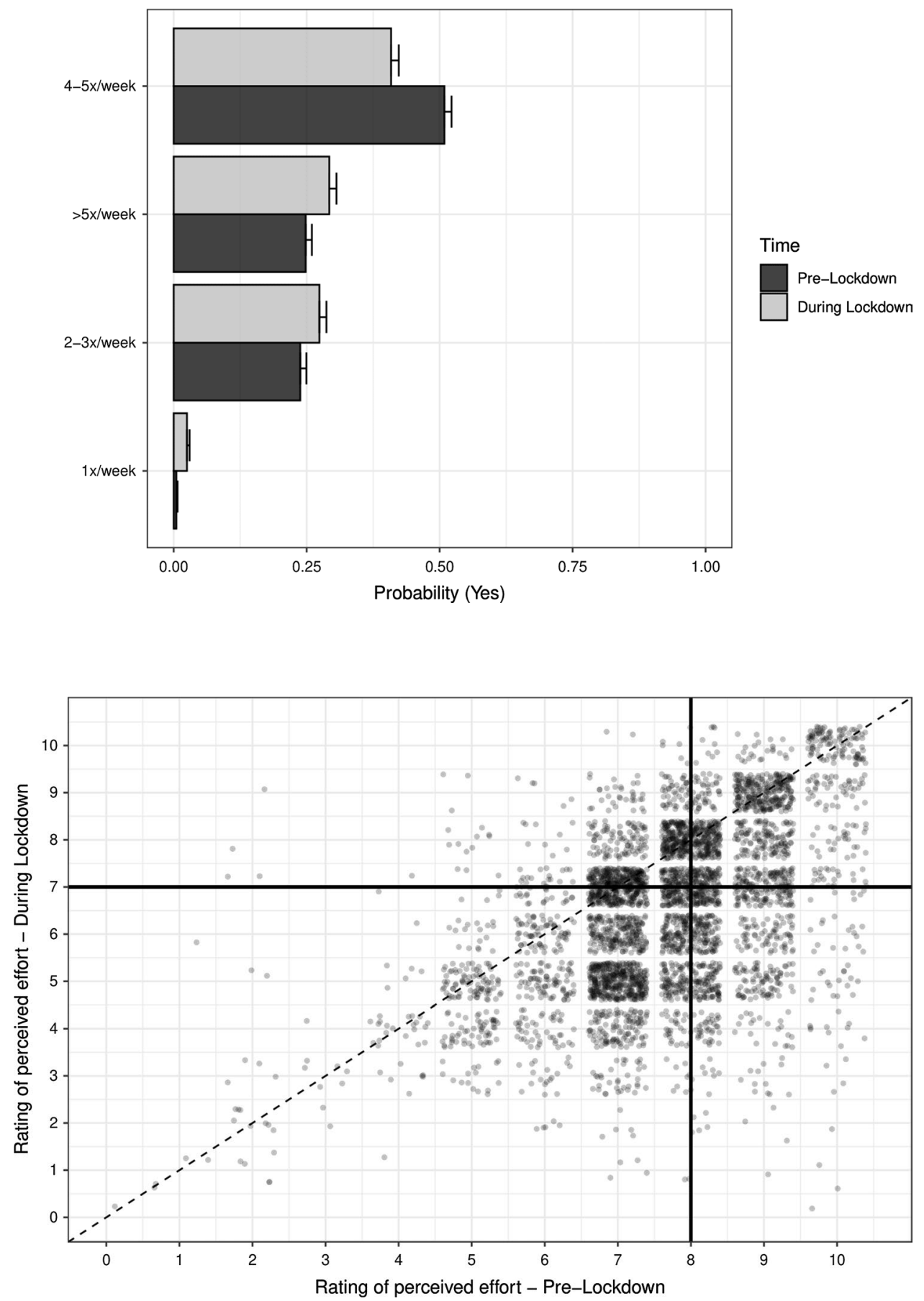

Rating perceived effort - Pre-Lockdown
Fig. 8 Scatter plot for "What was your usual intensity of perceived effort in your training?" over 'time' (i.e. pre- and during-lockdown). Note: the dotted diagonal line is the identity line for reference; the thick vertical and horizontal lines show the median values for 'pre-lockdown' and 'during lockdown,' respectively 'less effective' (38\%). For those who changed their goals, their current training was mostly perceived to be 'less effective' (54\%), though reported similar perceptions of effectiveness for their new goals with the majority reporting their training as 'similarly effective' (42\%).

\subsection{Exploratory Findings}

All exploratory plots are included in the supplementary files (S5 to S24) for readers to examine. We highlight only those exploratory findings that appear noteworthy here. 

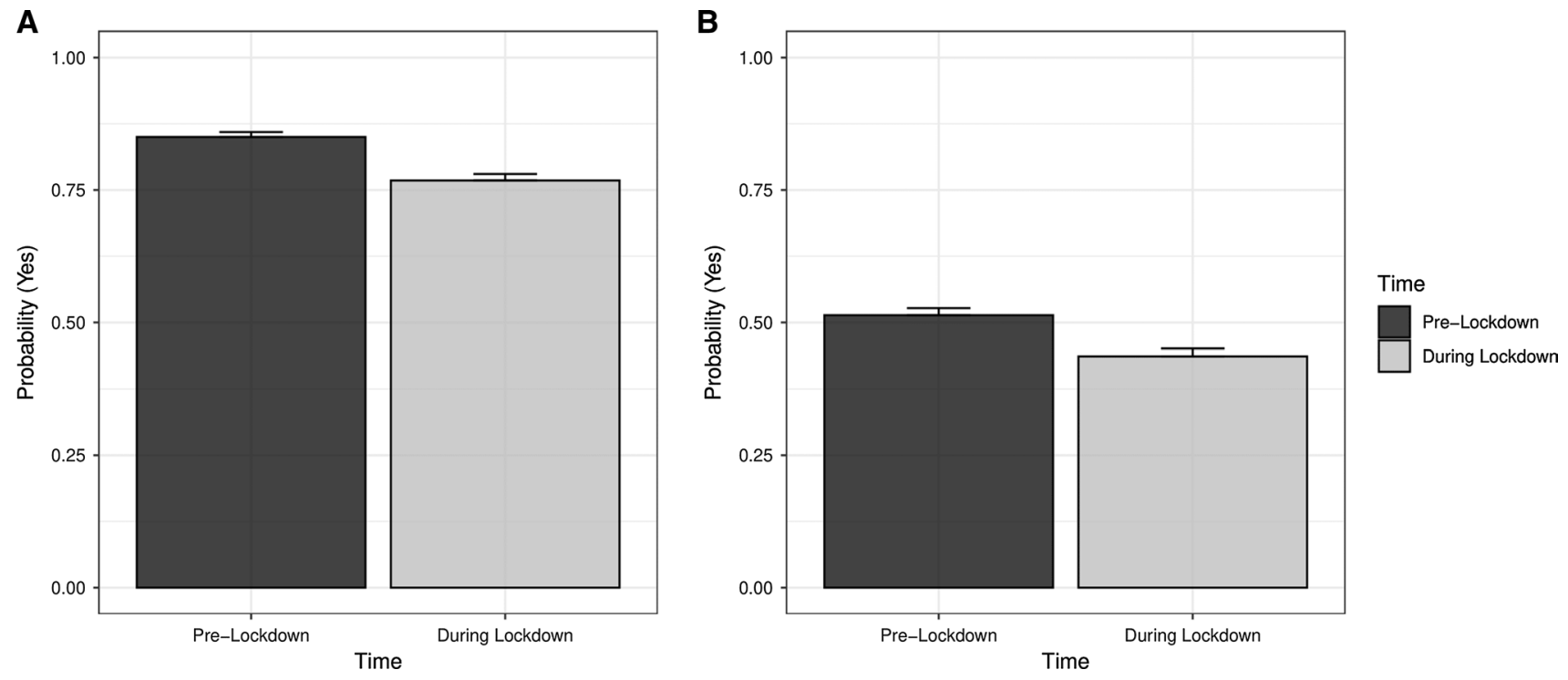

Fig. 9 Predicted marginal probabilities for “Did you usually plan/record each workout?" over 'time' (i.e. pre- and during-lockdown) and 'items'. Note: error bars are 95\% compatibility (confidence) intervals; $Y$ axis is ordered by the magnitude of probabilities at 'pre-lockdown'

Fig. 10 Predicted marginal probabilities for "Did you usually train in the morning, afternoon, evening or night-time?" over 'time' (i.e. pre- and duringlockdown) and 'items'. Note: error bars are $95 \%$ compatibility (confidence) intervals

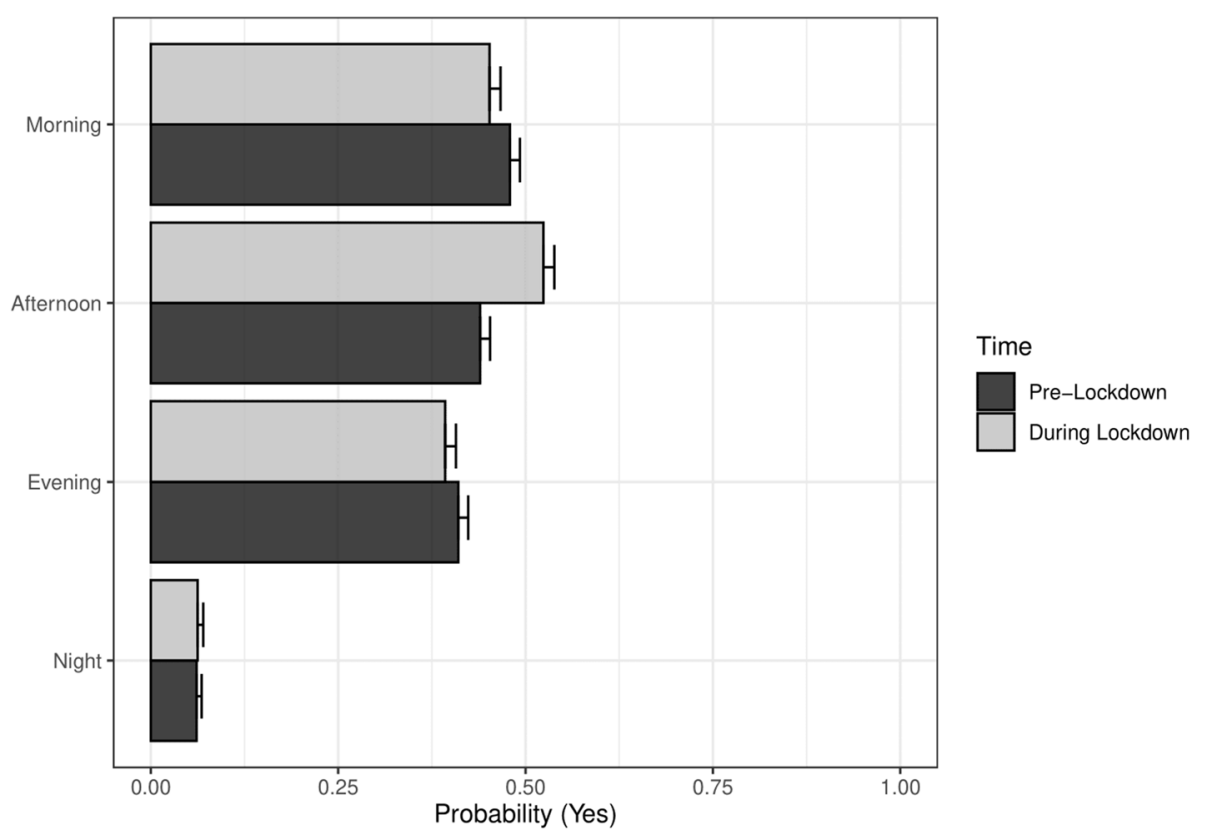

\subsubsection{Demographics Predictors of Training Behaviours}

Somewhat surprisingly, there was no clear impact of current motivation on probability of continuing RT. There were some suggestions of demographic predictors for the probability of continuing RT during-lockdown. A slightly greater probability was seen for those who have competed in sports (most clearly for strength sports and endurance sports participation), and a slightly greater probability of continuing with greater years of RT experience. However, across almost all predictors the probability of continuing RT remained fairly high ( 70-80\%). An exception was a lower probability of continuing for self-identified black respondents ( $60 \%$ ), yet due to the low sample number in this group the interval estimate is imprecise compared to other groups. Exploratory plots of probability of continuing RT are available in supplementary file S5.

There were few clear interactions between demographic predictors and probabilities of RT behaviours over time (i.e. pre- to during-lockdown) with most differences appearing minor. During-lockdown older individuals were slightly more likely to train 'supervised virtually/online' though the 


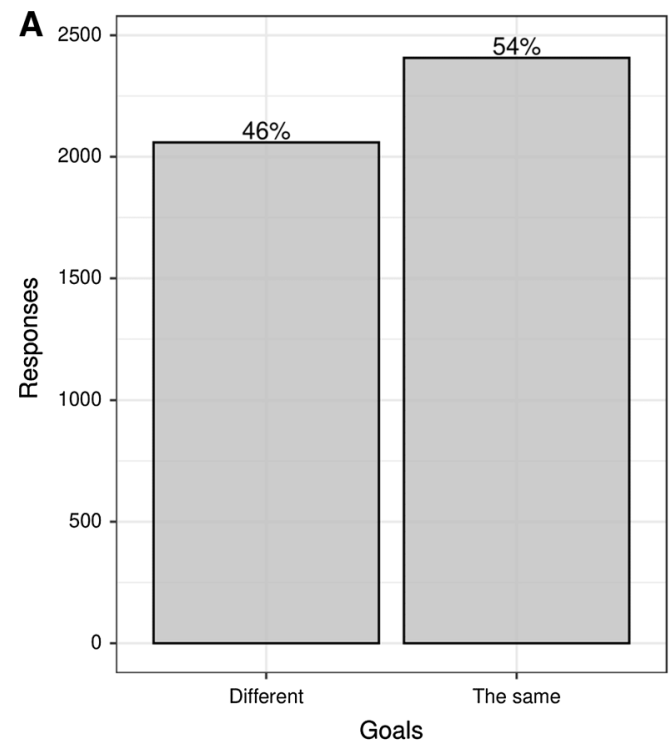

Fig. 11 a shows frequencies and exact marginal probabilities for the question "Are your goals for training different/the same?" as point estimates for questions relating to individuals training goals. b shows predicted marginal probabilities for "What are your goals from par-

probability was still low (supplementary file S8). There was a tendency towards greater probability of purchasing new equipment for those middle aged (supplementary file S7). Interestingly, those with greater RT experience were more likely to purchase equipment pre- lockdown, but less likely to do so during lockdown (supplementary file S7).

Some relationships between predictors and certain behaviours were consistent across both time points. This was most evident for the effects of age, RT experience, and goals; all other associations again appeared minor. With increased age individuals were less likely to train alone yet had increased probability of being 'supervised in person' and decreased probability of training 'with a partner(s)' (supplementary file S8). With increased age it was also more probable for people to perform full-body routines (supplementary file $S 9$ ) and be less likely to use free-weights but more likely to use resistance machines and perform cardio/aerobic exercise (supplementary file S10). Older individuals were also less likely to use lower repetitions ranges (i.e. 1-5 and 6-10 repetitions), were more likely to use $>10$ exercises and less likely to use 4-6 exercises, and also more likely to perform single sets of both exercises and for muscle groups (supplementary files S11 to S14). There was also a slight increase in probability of training $1 \mathrm{x} /$ week, a larger increase in training $2-3 \mathrm{x} /$ week, and similar decreases in 4-5 and $>5 \times$ /week with increased age (supplementary file S15). Older individuals were also more likely to train in the morning and less likely to train in the afternoon or evening (supplementary file S20), and their goals were more likely to be muscular strength, endurance, or general health, and less likely to be muscle growth, enjoyment, or social recognition (supplementary file S21). Interestingly, both weight and stress management as goals showed inverted U-shaped relationships with the greatest probabilities occurring during middle age (supplementary file S20). Many of the relationships with age were similar for RT experience likely due to their covariance. Goals were unsurprisingly associated with certain predictors; for example, having competed in strength sports, bodybuilding, and endurance sports were associated with greater probability of having muscular strength, growth, and endurance as goals (supplementary file S20). Those with higher BMI were also more likely to report weight management as a goal, men were more likely to report muscle growth, and women were more likely to report weight management, general health and stress management (supplementary file S20).

\subsubsection{Impact of RT Behaviours upon Perceived Effectiveness of Training, Enjoyment from Training, and Likelihood of Continuing Training}

With the exception of training frequency, there were not any apparent relationships between specific RT behaviours during lockdown and perceived effectiveness of training, enjoyment from training, and likelihood of continuing training (supplementary files S21 to S23). Those who were training $1 \mathrm{x} /$ week were somewhat more likely to perceive their training as less effective, less enjoyable (and those with higher frequencies reported more 
Fig. 12 Frequencies and proportions of responses for $\mathbf{a}$ motivation, $\mathbf{b}$ enjoyment, and $\mathbf{c}$ likelihood of continuing training

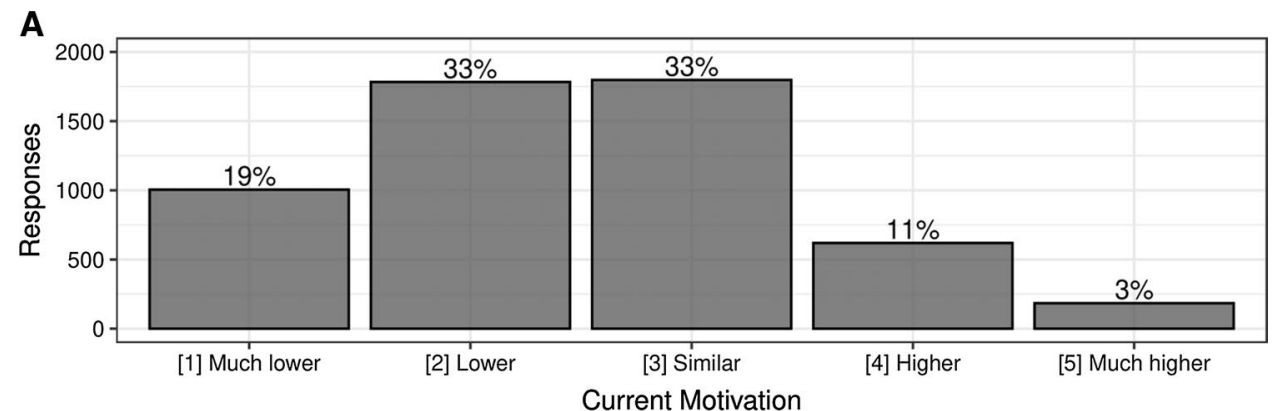

B

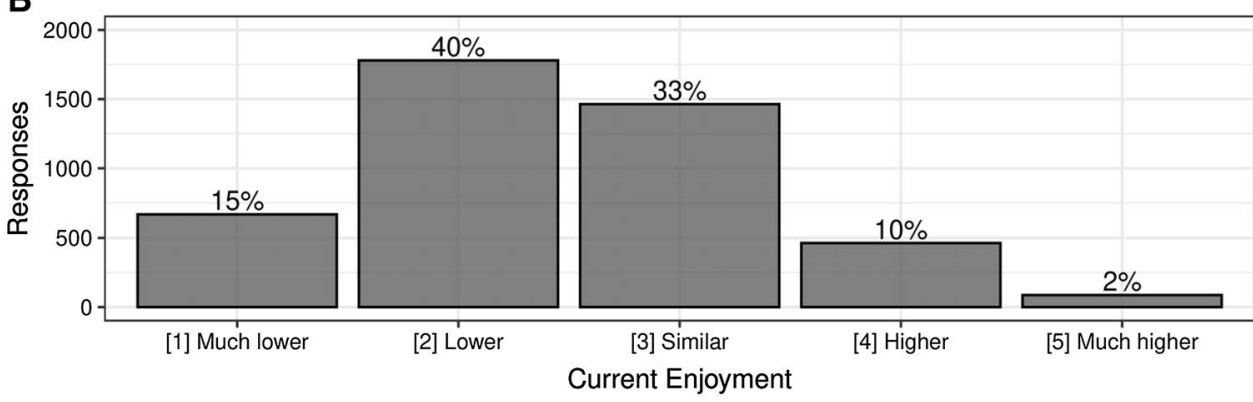

C

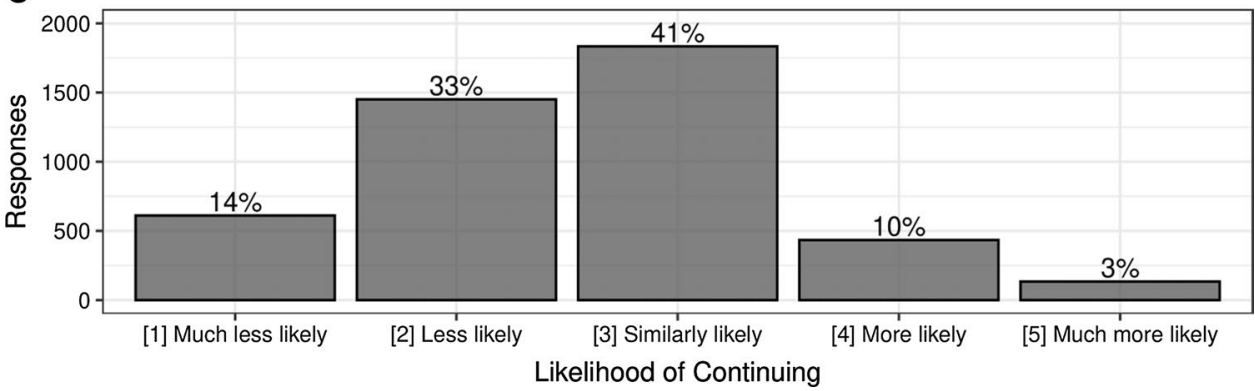

enjoyment), and were less likely to continue their current training. When looking at the breakdown across whether goals changed or not, for those who changed their goals during-lockdown current training was perceived to be less effective for both prior and current goals, and those who changed goals generally found training less enjoyable and were less likely to continue their current training.

\section{Discussion}

We found that the practice of RT, in those who previously self-identified as regularly engaging in RT, was largely unaffected (albeit with slight adaptations to certain behaviours) by various lockdown protocols that included closure of gyms and leisure facilities (i.e. the implementation of facility closures AND/OR social distancing). A majority of individuals $(82.8 \%)$ maintained participation in some form of RT during-lockdown and on the whole the probabilities of engaging in certain RT behaviours were largely similar (particularly numbers of exercises, sets per exercise or muscle group, and weekly frequencies). We did note some alterations in RT practice, however, including the following: a shift from primarily training in privately owned gyms to training at home; greater probability of training using a full-body routine; a reduction in use of resistance machines and an increase in free weight and bodyweight training; a reduction in use of moderate repetition ranges and greater use of higher repetition ranges; and a reduction in the perception of effort experienced during-training. Further, individuals were slightly less likely to plan or record training during-lockdown and many changed their training goals as a result of lockdown. For those who changed their training goals, their current training was perceived as less effective for their prior goals, though for their current goals it was mainly perceived as similarly effective as was training for those who did not change their goals. Overall people's motivation to train 
Fig. 13 Frequencies and proportions of responses for perceived effectiveness of current training for a current goals in those whose goals remained the same, b prior goals in those whose goals changed, and c current goals in those whose goals changed
A Note: Respondents whose goals remained the same

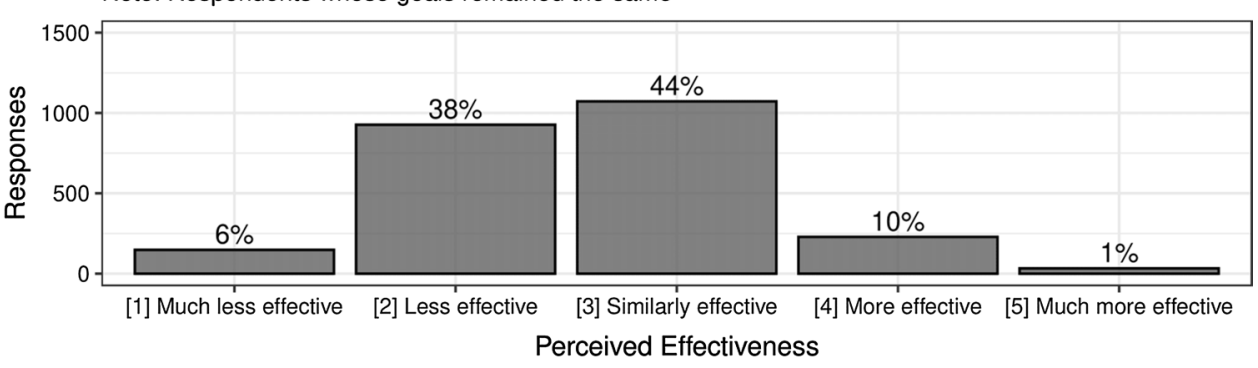

B Note: Respondents whose goals changed

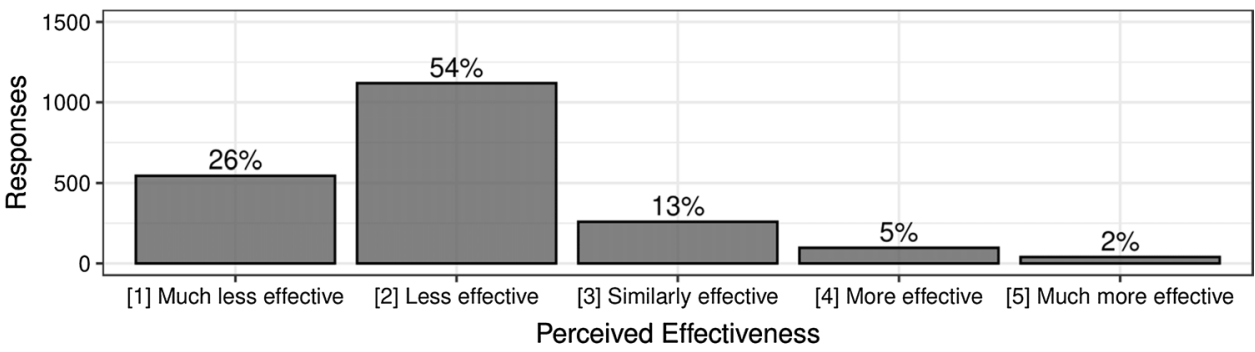

C Note: Respondents whose goals changed

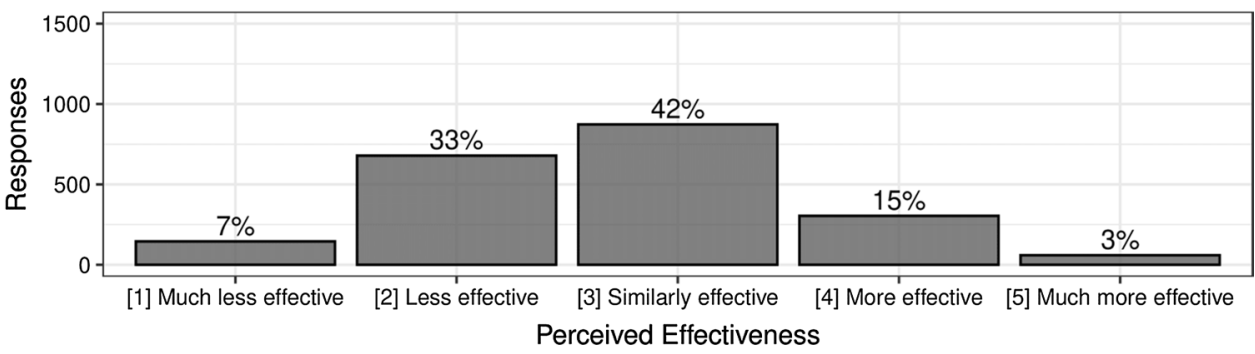

was similar, or lower; enjoyment from training was mostly lower; and people were similarly likely to continue with the training they were conducting during-lockdown. Interestingly, there were few obvious interactions of any demographic predictors and the impact of lockdown; however, there were some possible effects across time from age, RT experience, and training goals.

Those previously engaged in RT appeared largely able to maintain similar training behaviours (numbers of exercises, sets per exercise or muscle group, and weekly frequencies), yet adapted to performing their training within new locations and with alternate modalities. The shift to primarily homebased training is unsurprising given the facility closures and social distancing protocols implemented, and it may be that resistance training was more easily adapted to home environments compared to endurance training. Though the change in location may have implications such as loss of the typical psychosocial benefits of the gym environment [13], and also access to certain equipment (i.e. resistance machines), evidence suggests that a range of modalities of RT are broadly speaking similarly efficacious [34-37]. Free weight $^{10}$ and bodyweight exercises were the most common modality used during lockdown, in addition to a shift towards use of higher repetition ranges which may reflect the use of lower loads perhaps due to the difficulty of accessing and storing large amounts of free weights, in addition to the use of bodyweight exercise. Evidence suggests that both heavier- and lighter-load RT can produce similar adaptations in both general strength and muscular growth [34]; and further, when employed in a similar manner (as appears to have been done by respondents in this study), a variety

\footnotetext{
${ }^{10}$ Perhaps facilitated by many individuals already having access to such home equipment; as evidenced by a greater probability of having purchased equipment prior to lockdown. Based upon the wording of this question responses may in fact reflect those purchasing equipment in preparation for the coming lockdown to facilitate continued training at home. Indeed, those with more RT experience were more likely to purchase pre- lockdown, but less likely during-lockdown. Due to the surge in purchases of home-based equipment leading into and early on in lockdown, shortages were commonly reported duringlockdown rendering it difficult to make purchases during-that period and indeed after an initial spike demand dropped [17]. Those with greater RT experience may have better anticipated this.
} 
of modes of external resistance appear to produce largely similar outcomes [34-37]. Thus, although many perceived their training to be less effective during lockdown, evidence suggests that engagement was likely similarly efficacious.

Despite the relative stability of training behaviour, one clear difference in the manner in which RT was being performed was with respect to intensity of effort. On average there was a 'moderate' drop in rating of perceived effort based upon the standardised effect size (though this was only $1 \mathrm{pt}$ on the $11 \mathrm{pt}$ scale used). Studies of athletes during this period have reported similar reductions in perception of effort for training [38, 39]. This may have implications for the efficacy of RT given the important role that effort may play in determining outcomes $[2,40]$. This outcome may be explained, at least in part, by fears of immunosuppression and subsequent risk of infection considering the 'open window' theory and the dose-response impact of intensity of effort [24, 41].

Perhaps a more salient factor though may be motivation; indeed, current motivation for training was skewed towards being lower during lockdown. Motivation is thought to be a determinant of the intensity of a given behaviour engaged in (i.e. effort [42]) and an unplanned exploratory analysis of our data ${ }^{11}$ suggested there was a relationship between current motivation and the change in rating of perceived effort (i.e. during-minus pre- lockdown). Those who were more motivated were more likely to maintain their training effort.

Reporting of lower motivation for current training was accompanied by other corroborating factors such as a reduced likelihood of engaging in planning of RT behaviours. These effects could be explained under several theories. Within the Theory of Planned Behaviour, perceived behavioural control is thought to be a key component in determining engagement in exercise including RT [43]. Further, within Social Determination Theory there is evidence of consistent relationships between autonomous motivations, goals, and exercise engagement [44]. Indeed, choice and the ability to be autonomous has been proposed as important for successful engagement in RT, increasing enjoyment, adherence, and its benefits [45]. Notably, respondents typically reported lower enjoyment as well. The clearest impact upon perceived effectiveness, enjoyment, and likelihood of continuing training, however, was upon those who had changed their training goals. Frequency was also a factor in this regard, as those training $1 \mathrm{x} /$ week were somewhat more likely to perceive their training as less effective and less enjoyable, and were less likely to continue their current training. In contrast, those exercising at higher frequencies reported

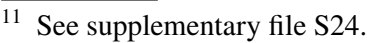

more enjoyment. Indeed, frequency appeared to be associated with motivation also in an unplanned exploratory analysis ${ }^{12}$ and thus lower engagement might have been a result of reduced motivation. Lockdown likely forced people to change their training habits thus impacting upon their perceptions of autonomy and control. This was most influential for those who also changed their training goals, perhaps through necessity of the enforced lockdown impacting behaviours, who felt that their new training was less effective for their previous goals and thus shifted those goals to something it would be effective for. It did not seem that any specific aspects of training behaviour changes were particularly influential. While previous work has indicated that a shift towards free weight use could increase enjoyment compared to resistance machines [46], we found no impact of modality of training on enjoyment. Thus, loss of control of training was likely the more impactful factor upon people's perceptions, intentions, and engagement.

The general resilience of RT behaviours to the lockdown warrants further examination of those who stopped training during-lockdown at follow-up to understand whether this was a temporary behaviour change, or longer term. Considering the Transtheoretical Model of behaviour change, recommencing a positive behaviour such as exercise is typically challenging after it is broken (i.e. 'relapse' [47]). However, though facilities have begun to reopen in many countries, reviews of consumer confidence surveys show varying intentions to return to using gyms [17]. Some evidence suggests that with new guidelines for hygiene and social distancing, gym reopening may not increase risk of COVID-19 spread [48]. Of the 923 individuals in our study who said they had stopped training, $663(72 \%)$ consented to be contacted for a follow up survey. Thus, future work will explore whether individuals in our survey who ceased RT return to participation.

\subsection{Strengths and Limitations}

A strength of this study was the speed with which we were able to implement the surveys (including translations) and the sample size achieved. However, the predominant demographic represented was white US males thus potentially affecting the generalisability of the findings. Indeed, a large proportion were also educated to University level in a topic related to physical activity, exercise, or sport which might be expected to facilitate resilience in RT behaviours. Further, relatively few were care-givers which would be expected to influence ability to maintain RT

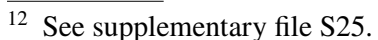


behaviours. History bias may be a potential concern in interpreting the effects of implementation of lockdown policies in pre- to during-analyses. However, our screening excluded those who were not currently subject to such measures and so imposed some control for the impact of varying policies between countries. One factor which was not controlled for, however, was the time since lockdown began and thus the potential for recall bias, or for short term changes in behaviour for those who had been under lockdown for longer periods (up to $\sim 5$ weeks). Though it could be considered a strength with respect to the population sampled (i.e. resistance trained individuals), sampling bias due to the distribution of the survey through the authors' social and professional networks may further limit the extent to which inferences can be generalised to the general population of those engaged in RT. Lastly, due to the urgency of responding to the implementation of lockdown, our survey was developed ad hoc and did not undergo any psychometric validation process. However, given the specific expertise of the research group in RT we felt confident in the face validity of the questions, particularly relating to relevant RT behaviours. As a research group we in essence followed, albeit informally, approaches to expert development and validation of surveys [49]. Further, although we were able to translate into several languages with the inclusion of an international multi-lingual research group, we did not have sufficient time to engage in more robust forward and backwards translation [50].

\section{Conclusions}

Those engaged in RT prior to the COVID-19 lockdown appeared mostly able to maintain these behaviours with only slight adaptations in both the location and types of training performed (i.e. home-based free weight and bodyweight training). However, people employed less effort, and motivation, perceived effectiveness, enjoyment, and likelihood of continuing current training all appeared lower during-lockdown which may have resulted from a loss of control and autonomy in goal selection and behavioural choices. These results have implications for strategies to maintain engagement in positive health behaviours such as RT during-restrictive pandemic-related public health measures. It is unclear what the long-term effects of lockdown will be upon behaviours (including for those who stopped training) and perceptions and so future work will follow-up the cohort in this study.
Supplementary Information The online version contains supplementary material available at https://doi.org/10.1007/ s40279-021-01438-5.

Author contributions JS, JPF, LC, BJS, SP, RW, DW, DS, and PA-K conceived and designed the study. TA, SD, MVF, FS, TRL, PG, TK, JG, MS, and AP contributed materials and translations or survey tools. JS performed the data cleaning and analysis. All authors interpreted the results. JS and BJS drafted the initial manuscript. All authors edited and finalised the manuscript.

Funding No funding was received in support of this research.

Availability of data and materials All data and materials are openly available on the Open Science Framework project page for this study (https://osf.io/xhryf/) and in the supplementary files.

Code availability All analysis code is openly available on the Open Science Framework project page for this study (https://osf. $\mathrm{io} / \mathrm{xhryf} /$ ) and in the supplementary files.

\section{Compliance with ethical standards}

Conflict of interest None of the authors have any conflicts of interest relevant to the subject matter of this manuscript to declare. Stuart Phillips reports grants from Canadian Institutes of Health Research, grants from National Science and Engineering Council, grant from the US NIH, grant from the Alliance for Potato Research and Education, grant from US National Dairy Council, outside the submitted work, in addition to: personal fees (honorarium) from Enhanced Recovery (donated to charity), the US National Dairy Council, the US Dairy Export Council, Leprino Foods and Agropur outside the submitted work.; travel costs from the US National Dairy Council, the US Dairy Export Council, and Leprino Foods and Agropur, outside the submitted work; equity in Exerkine (all proceeds donated to charity). Stuart Phillips is a named inventor on a patent 3052324 issued to Exerkine, a patent 16/182891 pending to Exerkine, and a patent 2018157258 pending to Exerkine.

Ethics approval This study received ethical approval from the Health, Exercise, and Sport Science Ethics Committee at Solent University (fishj2020).

Consent to participate Participants were provided with an information sheet containing all details of the study, and then were required to confirm they had understood this and provide informed consent to participate.

\section{References}

1. Maestroni L, Read P, Bishop C, Papadopoulos K, Suchomel TJ, Comfort P, Turner A. The benefits of strength training on musculoskeletal system health: practical applications for interdisciplinary care. Sports Med. 2020;50(8):1431-50.

2. Steele J, Fisher J, Skivington M, Dunn C, Arnold J, Tew G, Batterham AM, Nunan D, O'Driscoll JM, Mann S, Beedie C, Jobson S, Smith D, Vigotsky A, Phillips S, Estabrooks $\mathrm{P}$, Winett $\mathrm{R}$. A higher effort-based paradigm in physical activity and exercise for public health: making the case for a 
greater emphasis on resistance training. BMC Public Health. 2017;17(1):300.

3. World Health Organisation. Information sheet: global recommendations on physical activity for health 18-64 years old. 2011. http://www.who.int/dietphysicalactivity/publications/ recommendations18_64yearsold/en/. Accessed 1st Sep 2020.

4. US Department of Health and Human Services. Physical Activity Guidelines for Americans, 2nd ed. 2018. https:// health.gov/sites/default/files/2019-09/Physical_Activity_ Guidelines_2nd_edition.pdf. Accessed 1st Sep 2020.

5. UK Chief Medical Officers. Physical activity guidelines: UK Chief Medical Officers' report. 2019. https://www.gov. uk/government/publications/physical-activity-guidelines-ukchief-medical-officers-report. Accessed 1st September 2020.

6. Strain T, Fitzsimons C, Kelly P, Mutrie N. The forgotten guidelines: cross-sectional analysis of participation in muscle strengthening and balance \& co-ordination activities by adults and older adults in Scotland. BMC Public Health. 2016;16:1108.

7. Scholes S, Mindell J. Chapter 2: physical activity in adults. In: Craig R, Mindell J, editors. Health survey for England 2012 volume 1: health, social care and lifestyles. Leeds: Health and Social Care Information Centre; 2013.

8. Jones H, Millward P, Buraimo B. Department for Culture, Media and Sport. Adult participation in sport: analysis of the taking part survey. 2011. https://www.gov.uk/government/ uploads/system/uploads/attachment_data/file/137986/tp-adultparticipation-sport-analysis.pdf. Accessed 1st Sep 2020

9. Loustalot F, Carlson SA, Kruger J, Buchner DM, Fulton JE. Muscle strengthening activities and participation among adults in the United States. Res Q Exerc Sport. 2013;84:30-8.

10. Dalbo VJ, Czerepusko JB, Tucker PS, Kingsley MI, Moon JR, Young K, Scanlan AT. Not sending the message: a low prevalence of strength-based exercise participation in rural and regional Central Queensland. Aust J Rural Health. 2015;23:295-301.

11. Bennie JA, Pedisic Z, van Uffelen JGZ, Charity MJ, Harvey JT, Banting LK, Vergeer I, Biddle SJH, Eime RM. Pumping iron in Australia: Prevalence, trends and sociodemographic correlates of muscle strengthening activity participation from a national sample of 195,926 adults. PLoS ONE. 2016;11:e0153225.

12. Humphries B, Stanton R, Scanlan A, Duncan MJ. The prevalence and performance of resistance exercise training activities in an Australian population in relation to health authority guidelines. J Sci Med Sport. 2018;21:616-20.

13. Unger JB, Johnson CA. Social relationships and physical activity in health club members. Am J Health Promot. 1995;9(5):340-3.

14. Schroeder EC, Welk GJ, Franke WD, Lee DC. Associations of health club membership with physical activity and cardiovascular health. PLoS ONE. 2017;12(1):e0170471.

15. Smith L, Jacob L, Butler L, Schuch F, Barnett Y, Grabovac I, Veronese N, Caperchione C, Lopez-Sanchez GF, Meyer J, Abufaraj M. Prevalence and correlates of physical activity in a sample of UK adults observing social distancing during-the COVID-19 pandemic. BMJ Open Sport Exerc Med. 2020;6(1):e000850.

16. Ding D, del Pozo CB, Green MA, Bauman AE. Is the COVID19 lockdown nudging people to be more active: a big data analysis. Br J Sports Med. 2020. https://doi.org/10.1136/bjspo rts-2020-102575 (Online ahead of print).

17. ukactive. COVID-19 Impact Report: the fitness and leisure sector's path to recovery. ukactive, 4Global, and DataHub. 2020. https://www.ukactive.com/wp-content/uploads/2020/
05/ukactive-COVID-19-Impact-Report.pdf. Accessed 1st Sep 2020.

18. López-Bueno R, Calatayud J, Andersen LL, BalsalobreFernández C, Casaña J, Casajús JA, Smith L, López-Sánchez GF. Immediate Impact of the COVID-19 confinement on physical activity levels in Spanish adults. Sustainability. 2020;12(14):5708.

19. Tison GH, Avram R, Kuhar P, Abreau S, Marcus GM, Pletcher MJ, Olgin JE. Worldwide Effect of COVID-19 on Physical Activity: A Descriptive Study. Ann Intern Med. 2020. https:// doi.org/10.7326/M20-2665.

20. Faulkner J, O'Brien W, McGrane B, Wadsworth D, Batten J, Askew CD, Badenhorst C, Byrd E, Coulter M, Draper N, Elliot C. Physical activity, mental health and well-being of adults during-early COVID-19 containment strategies: a multi-country cross-sectional analysis. medRxiv. 2020. https://doi.org/10. 1101/2020.07.15.20153791.

21. Jakobsson J, Malm C, Furberg F, Ekelund U, Svensson M. Physical activity during-the Coronavirus (COVID-19) pandemic: prevention of a decline in metabolic and immunological functions. Front Sport Act Living. 2020;2:57.

22. Hammami A, Harrabi B, Mohr M, Krustrup P. Physical activity and coronavirus disease 2019 (COVID-19): specific recommendations for home-based physical training. Manag Sport Leisure. 2019. https://doi.org/10.1080/23750472.2020.17574 94 (Online ahead of print).

23. Gentil P, Ramirez-Campillo R, Souza D. RT in face of the Coronavirus outbreak: time to think outside the box. Front Physiol. 2020;11:859.

24. Ferreira-Júnior JB, Freitas EDS, Chaves SFN. Exercise: a protective measures or an "Open Window" for COVID-19? A mini review. Front Sports Act Living. 2020;2:51.

25. Campos MVA, Miguel H. Elastic RT: resistance exercise alternative in the home environment during-Covid-19 pandemic. Interam J Med Health. 2020;3:e202003006.

26. Latella C, Haff G. Global challenges of being a strength athlete during-a pandemic: impacts and sports-specific training considerations and recommendations. Sports. 2020;8:100.

27. Jukic I, Calleja-González J, Cos F, Cuzzolin F, Olmo J, Terrados N, Njaradi N, Sassi R, Requena B, Milanovic L, Krakan I, Chatzichristos, Alcaraz PE. Strategies and solutions for team sports athletes in isolation due to COVID-19. Sports. 2020;8:56

28. Toredashi BR, Asif IM. Coronavirus disease 2019 (COVID19): considerations for the competitive athlete. Sports Health. 2020;12(3):221-4

29. Steele J, Fisher J, McKinnon S, McKinnon P. Differentiation between perceived effort and discomfort during resistance training in older adults: reliability of trainee ratings of effort and discomfort, and reliability and validity of trainer ratings of trainee effort. J Trainol. 2017;6(1):1-8.

30. Amrhein V, Greenland S, McShane B. Scientists rise up against statistical significance. Nature. 2019;567:305-7.

31. McShane BB, Gal D, Gelman A, Robert C, Tackett JL. Abandon statistical significance. Am Stat. 2019;73:235-45.

32. Suesse TF, Liu I. Modelling strategies for repeated multiple response data. In: Centre for Statistical and Survey Methodology, University of Wollongong, Working Paper. 2011. https:// ro.uow.edu.au/cgi/viewcontent.cgi? article $=1074 \&$ context $=$ cssmwp. Accessed 1st Sep 2020.

33. Halekoh U, Højsgaard S, Yan J. The R package geepack for generalisaed estimating equations. J Stat Softw. 2006;15(2):1-11.

34. Schoenfeld BJ, Grgic J, Ogborn D, Krieger JW. Strength and hypertrophy adaptations between low- vs. high-load RT: a 
systematic review and meta-analysis. J Strength Cond Res. 2017;31(12):3508-23.

35. Kikuchi N, Nakazato K. Low load bench press and push up induce similar muscle hypertrophy and strength gain. J Exerc Sci Fit. 2017;15(1):37-42.

36. Kotarsky CJ, Christensen BK, Miller JS, Hackney KJ. Effect of progressive callisthenic push-up training on muscle strength and thickness. J Strength Cond Res. 2018;32(3):651-9.

37. Rossi FE, Schoenfeld BJ, Ocetnik S, et al. Strength, body composition, and functional outcomes in the squat versus leg press exercises. J Sports Med Phys Fit. 2018;58(3):263-70.

38. Mon-López D, de la Rubia RA, Hontoria Galán M, Refoyo RI. The impact of Covid-19 and the effect of psychological factors on training conditions of Handball Players. Int J Environ Res Public Health. 2020;17(18):6471.

39. Mon-López D, García-Aliaga A, Ginés Bartolomé A, Muriarte SD. How has COVID-19 modified training and mood in professional and non-professional football players? Physiol Behav. 2020;227:113148.

40. Morton RW, Colenso-Semple L, Phillips SM. Training for strength and hypertrophy: an evidence-based approach. Curr Opin Physiol. 2019;10:90-5.

41. Rahmati-Ahmadabad S, Hosseini F. Exercise against SARSCoV-2 (COVID-19): does workout intensity matter? (A mini review of some indirect evidence related to obesity). Obes Med. 2020;19:100245.

42. Inzlicht M, Shenhav A, Olivola CY. The effort paradox: effort is both costly and valued. Trends Cogn Sci. 2018;22(4):337-49.
43. Castellanos DC, Daprano CM, Blevins C, Crecelius A. The theory of planned behavior and strength training in collegeaged women. J Am Coll Health. 2020. https://doi.org/10.1080/ 07448481.2020 .1775606 (Online ahead of print).

44. Teixeira PJ, Carraca EV, Markland D, Silva MN, Ryan RM. Exercise, physical activity, and self-determination theory: a systematic review. Int J Behav Nutr Phys Act. 2012;9:78.

45. Halperin I, Wulf G, Vigotsky AD, Schoenfeld BJ, Behm DG. Autonomy: a missing ingredient of a successful program? Strength Cond J. 2018;40(4):18-25.

46. Carraro A, Paoli A, Gobbi E. Affective response to acute resistance exercise: a comparison among machines and free weights. Sport Sci Health. 2018;14(2):283-8.

47. Prochaska JO, Marcus BH. The transtheoretical model: Applications to exercise. In: Dishman RK, editor. Advances in exercise adherence. Human Kinetics Publishers; 1994. pp. 161-80.

48. The TRAiN Study Group. Randomized re-opening of training facilities during-the COVID-19 pandemic. medRxiv. 2020. https://doi.org/10.1101/2020.06.24.20138768

49. Artino AR Jr, La Rochelle JS, Dezee KJ, Gehlbach H. Developing questionnaires for educational research: AMEE Guide No. 87. Med Teach. 2014;36(6):463-74.

50. Tsang S, Royse CF, Terkawi AS. Guidelines for developing, translating, and validating a questionnaire in perioperative and pain medicine. Saudi J Anaesth. 2017;11(Suppl 1):S80-9. 


\section{Authors and Affiliations}

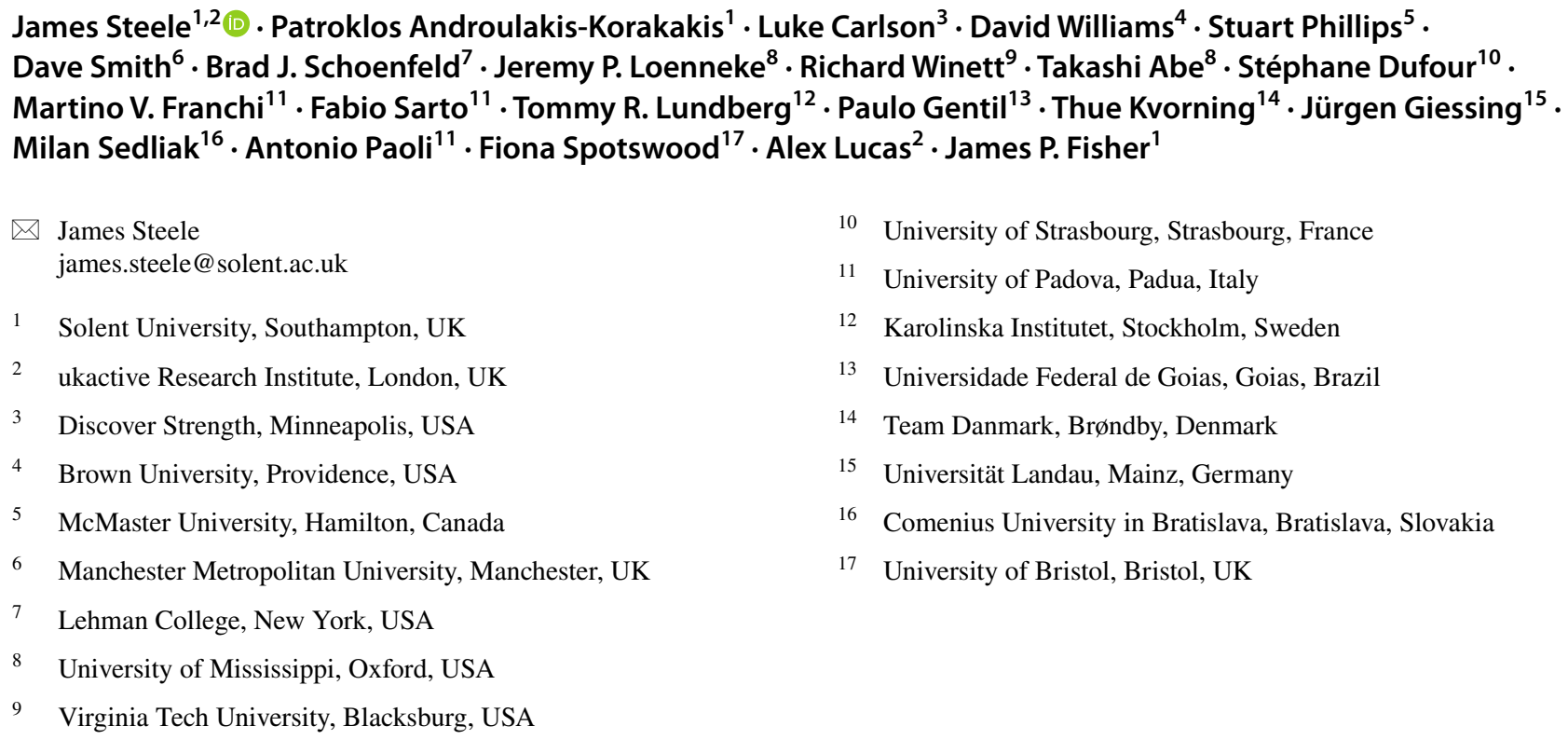

James Steele

james.steele@solent.ac.uk

Solent University, Southampton, UK

2 ukactive Research Institute, London, UK

3 Discover Strength, Minneapolis, USA

4 Brown University, Providence, USA

5 McMaster University, Hamilton, Canada

6 Manchester Metropolitan University, Manchester, UK

7 Lehman College, New York, USA

8 University of Mississippi, Oxford, USA

9 Virginia Tech University, Blacksburg, USA

10 University of Strasbourg, Strasbourg, France

11 University of Padova, Padua, Italy

12 Karolinska Institutet, Stockholm, Sweden

13 Universidade Federal de Goias, Goias, Brazil

14 Team Danmark, Brøndby, Denmark

15 Universität Landau, Mainz, Germany

16 Comenius University in Bratislava, Bratislava, Slovakia

17 University of Bristol, Bristol, UK 